\title{
Identification of Paediatric Tuberculosis from Airway Shape Features
}

\author{
Benjamin Irving ${ }^{1}$, Pierre Goussard ${ }^{2}$, Robert Gie ${ }^{2}$, Andrew Todd-Pokropek ${ }^{1}$, \\ and Paul Taylor ${ }^{1}$ \\ 1 University College London, London WC1E 6BT, UK \\ b.irving@ucl.ac.uk \\ 2 Tygerberg Hospital, Stellenbosch University, \\ Western Cape 7530, South Africa
}

\begin{abstract}
Clinical signs of paediatric pulmonary tuberculosis (TB) include stenosis and deformation of the airways. This paper presents two methods to analyse airway shape and detect airway pathology from CT images. Features were extracted using (1) the principal components of the airway surface mesh and (2) branch radius and orientation features. These methods were applied to a dataset of 61 TB and non-TB paediatric patients. Nested cross-validation of the support vector classifier found the sensitivity of detecting TB to be $86 \%$ and a specificity of $91 \%$ for the first 10 PCA modes while radius based features had a sensitivity of $86 \%$ and a specificity of $94 \%$. These methods show the potential of computer assisted detection of TB and other airway pathology from airway shape deformation.
\end{abstract}

\section{Introduction}

The prevalence of tuberculosis (TB) remains high in many developing countries while the accuracy of paediatric TB detection is low, and a combination of tests including imaging is used. Automated airway analysis has the potential to improve the detection of airway pathology such as TB. A common sign of primary TB in children is airway deformation caused by lymphadenopathy [1]. This can take the form of displacement and stenosis of airway branches, and widening of the carinal angle [1. Hila, mediastinal, subcarinal and paratracheal lymph nodes are commonly affected and the most common sites for compression are: the trachea, left main bronchus (LMB), right main bronchus (RMB) and bronchus intermedius (BI) 1]. This sign is more sensitive in children because the airways are more malleable and primary TB tends to affect the lymph nodes. Lymphadenopathy can also indicate other pathology but is useful for detecting $\mathrm{TB}$ when used in conjunction with other tests and is likely to indicate TB in areas with a high TB prevalence.

Paediatric airways are considerably smaller than those of adult patients, which means a lower resolution using the same voxel size, and fewer branches can be identified. Movement artefacts are also more likely because it is not possible to perform a breath hold scan on infants 10 .

G. Fichtinger, A. Martel, and T. Peters (Eds.): MICCAI 2011, Part III, LNCS 6893, pp. 133-140, 2011.

(C) Springer-Verlag Berlin Heidelberg 2011 
Point distribution models (PDM) are a common method of modelling shape variation. Anatomical landmarks or a mesh are used to represent a shape and the variation in position of corresponding points is calculated. Principal component analysis (PCA) can be applied to the PDM to reduce the dimensionality of the representation, identifying the principal modes of variation. These techniques have been applied successfully in a number of cases including facial morphology [5]. However, very little research has focussed on airway shape modelling. A previous study developing a shape model of the airways focussed on patient specific models and required manual interaction [4].

An alternative and more intuitive approach is to use features that correspond directly to clinical observations but this requires more background knowledge. In this paper we present a complete system for analysing pathological airway shape variation and compare two approaches for identifying TB cases: one using features generated using the principal modes of variation of a surface mesh, and another using features based on the branch radius and orientation. We test both on a dataset of TB and non-TB cases. Contributions of this work include a method to generate a shape model of normal and pathological airway variation (the authors are not aware of any previous method to model airway pathology and particularly to distinguish between TB and non-TB datasets) and methods focussed on paediatric datasets. Additional contributions include a novel method for automatically generating airway landmarks based on the airway topology and centreline, extension of mesh-warping to suit stenosed airway shape variation and the training of a classifier on airway shape data.

\section{Method}

An automated airway segmentation approach was used and the centreline and bifurcation points extracted. Corresponding landmark points were generated and a template mesh was warped to each airway. A shape model was then developed using the principal modes of the corresponding vertices. Cross-section diameter measurements were made for each branch and used to generate the second feature set.

\subsection{Dataset and Airway Extraction}

The dataset used in this study consists of TB and non-TB cases. 29 chest CT scans of paediatric patients diagnosed with definite or probable TB from a positive culture, or bronchoscopy and CT findings were acquired from Tygerberg Hospital in South Africa (mean age $22 \pm 26$ months) and 32 chest CT scans of paediatric patients with a non-TB diagnosis were acquired from Gt Ormond St Hospital, London (mean age $38 \pm 22$ months). Voxel size in the axial plane ranged from $0.3-0.5 \mathrm{~mm}$ and slice thickness $0.7-1 \mathrm{~mm}$. 13 cases with completely obstructed branches were previously manually excluded from the dataset because these cases can be easily identified and are not of interest for building a shape model. The age difference between the groups is within one standard deviation and age does not influence airway proportions in children [8]. 
The airways were segmented using an existing method [6] . This method uses morphological closing and reconstruction to enhance possible airway locations in the axial, coronal and sagittal directions. A region growing method, seeded at the trachea, is then used to extract the airway region. The structure of the airways is found using centreline extraction, branch point detection and branch labelling. Palágyi et al. [9]'s skeletonisation method is used for the extraction of the centreline because of its previous application to the airways. This is an iterative thinning approach, where each surface voxel is analysed in terms of orientation and connectivity and simple points are iteratively removed.

False branching can occur because of surface deformation (particularly when pathology is present) and, therefore, branch pruning is required. We found that false branches connected to the trachea, LMB and RMB can be longer than true branches further down the tree, and false branches may bifurcate. Therefore, a multilevel pruning system was developed that removed branches less than a specified length $(l)$ and removed larger false branches associated with the primary branches and smaller false branches associated with later generations. Three pruned trees $\left(T_{l_{1}}, T_{l_{2}}, T_{l_{3}}\right)$ were created with pruning $l_{1}>l_{2}>l_{3}$. A final tree was constructed from $T_{l_{1}}$ for the trachea, $T_{l_{2}}$ for the LMB and RMB and from $T_{l_{3}}$ for the remaining branches. A one voxel thick centre line was used to identify the branching structure, shown in Figure 1, a branch point was defined as a point with three neighbours in the $3 \times 3 \times 3$ surrounding region.

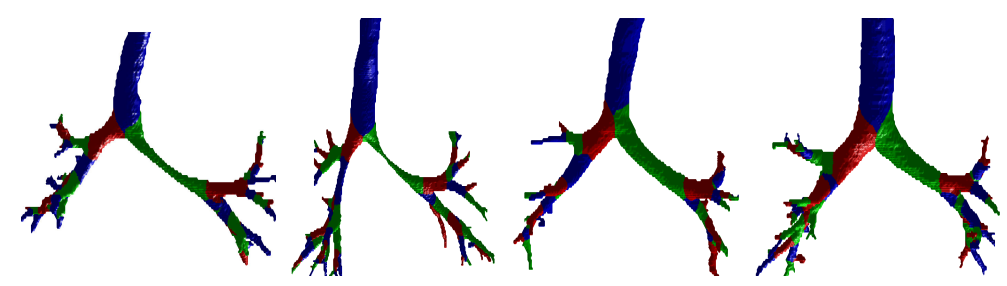

Fig. 1. Paediatric airway segmentation and branch-point identification. The two cases on the left show signs of TB while the others are non-TB cases.

\subsection{Corresponding Surface Point Generation and Mesh Alignment}

Surface point correspondence is required to derive features from a shape model while diameter based features require only regular sampling of the branch. Branch points are the only major anatomical landmarks and, therefore, corresponding points were generated by calculating the intersection between the surface and vectors orthogonal to the smoothed centreline at equidistant positions along each branch (Figure 22). The generated points take into account branch topology, medial line curvature and surface deformation. The analysis was performed on the trachea, RMB and LMB (commonly deformed by lymphadenopathy).

As discussed earlier, two sets of features are being considered, the principal modes of the surface deformation and branch radius/direction based features. 


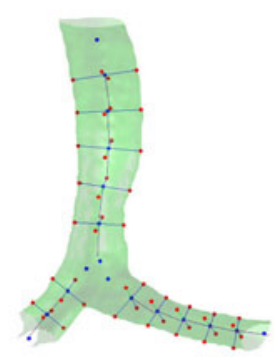

Fig. 2. Surface point placement using the centreline and bifurcation points

The surface points were used to calculate the two orthogonal diameters at each cross section along each branch. These points were generated from 60 equidistant points on the medial line of the trachea, 50 along the LMB and 30 along the RMB. A subset of the corresponding points (generated from 5 equidistant points on the Trachea and LMB, and 2 on the RMB) were used to warp a mesh onto each airway using Thin Plate Spline (TPS) warp. TPS warping is a common method of aligning objects using a set of landmark points 5. TPS attempts to perform realistic deformation by minimising the bending energy 2. The TPS function that minimises the energy is:

$$
f_{j}\left(P_{j}\right)=\sum_{i=1}^{k} w_{i j} U\left(P_{j}-P_{i j}\right)+a_{0}+a_{x} x+a_{y} y+a_{z} z
$$

where $f$ is the new position of the point and $f_{j}$ is a component of $\mathrm{f}, j \in(x, y, z)$, $P$ are the landmark points on the shape and $w_{i j}$ are the weighting factors. $w_{i j}$ can be found from the corresponding landmark points.

Further matching is required so that the template mesh is aligned with each target mesh (as shown in Figure 3). The simplest method is to project the template mesh to the closest point on the target mesh [5] but this can lead to unrealistic deformation while not covering small deformations. Figure 4 shows this mesh misalignment because of narrow sections caused by stenosis and the proposed solution.

Kaus et al 7 optimise the fit based on the distance between the meshes while an additional force preserves the mesh structure. We add a third term based on surface orientation. For each vertex on the template mesh $\left(t_{i}\right)$, a force $\left(F_{i, t o t}\right)$ is calculated to direct the warp. The closest point $\left(r_{i}\right)$ on the object mesh component is included to align the meshes (Eqn 2) and an internal forcing component is included to preserve the size of the faces (change in the distance of each of the $p$ neighbouring vertices to a vertex $t_{i}$ from the initial distance $v_{0 j}$ ) (Eqn 3). An expansion/contraction force is also added, based on the normal of each vertex $\hat{n}_{i}$ (calculated from the normal of the surrounding faces) controlled by the distance and direction of the target mesh to $F_{i, 1}$ (Eqn (4). This improves performance for small surface indentations/protrusions associated with stenosis. 


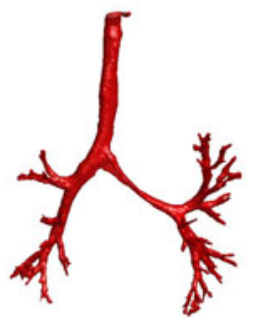

(a) Template image

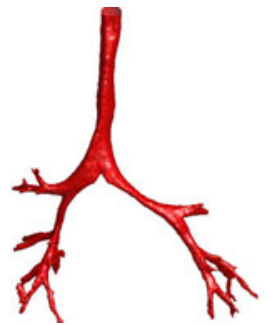

(b) Case to be matched

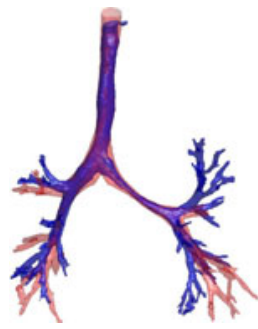

(c) TPS warp

Fig. 3. TPS warp using landmarks on the trachea, LMB and RMB

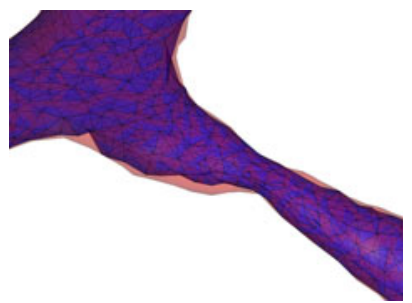

(a) Matching to the closest point

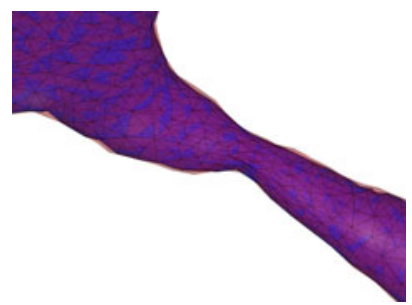

(b) Meshing procedure using $F_{1}, F_{2}$ and $F_{3}$

Fig. 4. Mesh matching

$$
\begin{array}{lr}
F_{i, 1}=\boldsymbol{r}_{\boldsymbol{i}}-\boldsymbol{t}_{\boldsymbol{i}} & \text { nearest point ext. force } \\
F_{i, 2}=\sum_{j}^{p} \hat{v}_{j}\left(\left\|\boldsymbol{v}_{\boldsymbol{j}}\right\|-\left\|\boldsymbol{v}_{\mathbf{0} \boldsymbol{j}}\right\|\right) \text { where } \boldsymbol{v}_{\boldsymbol{j}}=\boldsymbol{t}_{\boldsymbol{j}}-\boldsymbol{t}_{\boldsymbol{i}} & \text { internal force } \\
F_{i, 3}=\hat{n}_{i}\left(\hat{n}_{i} \cdot F_{i, 1}\right) & \text { normal ext. force } \\
F_{i, t o t}=\alpha F_{i, 1}+\beta F_{i, 2}+\gamma F_{i, 3} &
\end{array}
$$

In Equation 5, the forces are weighted with $\alpha, \beta$ and $\gamma$. This procedure is applied iteratively until stability is reached.

\subsection{Feature Extraction and Classification}

Each shape is represented as a $3 n$ dimensional vector where $n$ is the number of vertices in the mesh; $n \approx 1500$ was used in this study. Each shape was aligned using Generalised Procrustes analysis and PCA was applied to reduce the dimensionality and obtain a set of features for classification. PCA applies a linear transform that projects the PDM onto an uncorrelated space and can be used to extract relevant features [3. PCA modes are ordered by the variance and, therefore, can be used to reduce the dimensionality of the feature vector. For PCA, it can be shown that the eigenvectors of the covariance matrix 
$\sum=X X^{T}$ (where each column of $\mathrm{X}$ is a $3 n$ vector for each airway) can be used to project the dataset into the uncorrelated space $(b)$ represented by the eigenvectors $\boldsymbol{b}=\Phi^{T}(\boldsymbol{x}-\overline{\boldsymbol{x}})$ where the projection matrix $\left(\Phi^{T}\right)$ is the transpose of the eigenvector matrix $(\Phi)$. Therefore, a measurement vector $\boldsymbol{x}=\overline{\boldsymbol{x}}+\Phi \boldsymbol{b}$ can be represented in terms of the mean and displacement along each mode [3].

Three radius based features were calculated for each branch: the maximum ratio of the orthogonal diameters for each branch $\left(\max \left(\frac{d 1}{d 2}\right)\right)$, the ratio between the branch length and average branch diameter $\left(\frac{d_{a v e}}{l}\right)$ and the maximum ratio of local minima and neighbouring local maxima of the diameter as a function of position on the branch $\left(\frac{l \max 1+\operatorname{lmax} x_{2}}{2 l \min }\right)$. These features, based on advice from our clinical partners, were used as indicators of branch circularity, thickness and local stenosis, and were calculated for the trachea, RMB and LMB. The carinal angle was also calculated for each airway by fitting a line to the first third of the RMB and LMB and calculating the angle from bifurcation. All features were normalised.

Once a set of features was found to represent each airway in the dataset, a classifier was trained to distinguish between TB and non-TB cases. A Support Vector Machine (SVM) was chosen as the classifier because of its suitability for small datasets and the PRtools implementation of SVM was used. Leave-one-out cross validation (LOOCV) and nested CV were used to evaluate the classifier.

\section{Results}

Parameters for the mesh warp $\alpha, \beta$ and $\gamma$ were determined by comparing the volume generated from both the template mesh and the original mesh $\left(V_{\text {dif }}=\right.$ $\left(V_{\text {temp }} \backslash V_{\text {case }}\right) \cup\left(V_{\text {case }} \backslash V_{\text {temp }}\right)$. In order to focus on local errors instead of differences due to mesh face sizes, a morphological closing was applied $\left(V_{\text {open }}=\right.$ $V_{\text {dif }} \circ K$ where $\mathrm{K}$ is 6-connected kernel) in order to remove 1-voxel thick errors but retain larger local errors. Optimum parameters are around $\alpha=0.2$ and $\gamma=1$, where proportion of general error (without closing) is less than 0.022 and local error (with closing) is less than 0.002. Without the expansion force $(\gamma=0)$ then the minimum errors are 0.05 and 0.02 respectively. Fixed parameters were used for the whole dataset but could be chosen for each individual airway.

The SVM classifier was trained and tested on the two sets of features. Classification using PCA features were performed using the first 10 modes which represented $90 \%$ of the shape variation. Figure 5 shows the mean and variation from $-3 \sqrt{\lambda_{i}}$ to $3 \sqrt{\lambda_{i}}$ along the first 4 modes. Classification was also performed on the 10 radius and orientation based features. This classifier was optimised by adjusting the "trade-off parameter" $C$ (between 5 and 500) and the degree of the polynomial kernel (between 1 and 13) while running LOOCV for each choice. These values were chosen to cover a reasonable range of parameters but further optimisation could be performed. LOOCV was used because the dataset was too small to divide into a testing and training set. However, adjusting the SVM parameters with LOOCV allows the best classifier to be selected but can lead to a biased measure of accuracy. Therefore, to determine an unbiased sensitivity 
Table 1. Sensitivity and specificity using (1) the PCA and (2) the radius and orientation based feature set with LOOCV and Nested CV

\begin{tabular}{lll|ll} 
& \multicolumn{2}{c}{ LOOCV } & \multicolumn{2}{c}{ Nested CV } \\
& PCA & Rad & PCA Rad \\
\hline Sensitivity & $93 \%$ & $93 \%$ & $86 \%$ & $86 \%$ \\
Specificity & $94 \%$ & $94 \%$ & $91 \%$ & $94 \%$ \\
\hline
\end{tabular}

Mode 1

Mode 3

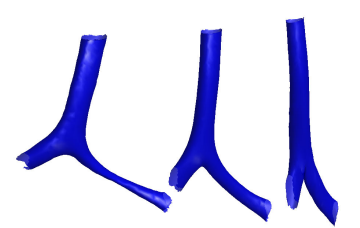

Mode 2

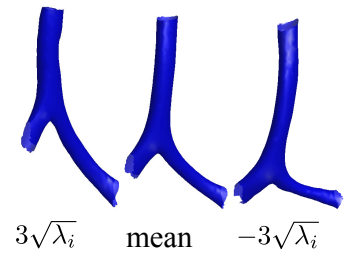

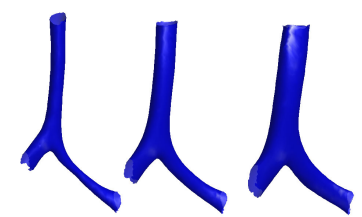

Mode 4

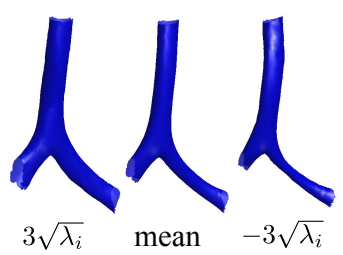

Fig. 5. Variation along the first four PCA modes

and specificity without an independent training set, nested CV was used [1]. Nested CV includes a second LOOCV loop with parameter optimisation inside the full LOOCV loop and results have been shown to be close to that of an independent testing set [11. Using LOOCV, the classifiers performed the same and parameters of $\mathrm{C}=100$ and 3 and polynomial degree of 3 and 1 were found for the PCA and radius based classifiers, respectively (Table 1). The radius based features performed slightly better when tested using nested CV (6 compared to 7 misclassified out of 61 ). The software was written in Matlab and $\mathrm{C}++$ and tested on a $2.0 \mathrm{GHz}$ quad-core processor. Generation of features from a segmented dataset and cross validation: $\approx 700$ s for the PCA based feature vector and $\approx 1200$ s for the radius based feature vector.

\section{Discussion}

In this paper we discuss two methods to quantify and detect airway shape deformation due to TB. Both these methods were able accurately to distinguish between paediatric cases with TB and without TB, and demonstrate the potential of these techniques to assist in the detection of airway pathology. PCA based features may be more generalizable and, more effective for differentiating other types of pathology without adjusting the feature choice. 
The datasets were collected from two hospitals and it is possible that population differences also have an effect on the classification. However, the features extracted using PCA correspond to clinical signs of TB. Examining Figure 5. the modes correspond to stenosis and widening of the carinal angle, which is consistent with clinical signs of TB [1]. The other feature set was based on characteristics of airway pathology.

This paper shows the potential of automated airway analysis to assist in the identification of pathology with possible CAD applications. The model could be developed further by training on localised pathology, or applied to other areas such as airway deformation and narrowing caused by congenital cardiac disease.

\section{References}

1. Andronikou, S., Wieselthaler, N.: Modern imaging of tuberculosis in children: thoracic, central nervous system and abdominal tuberculosis. Pediatr. Radiol. 34, 861875 (2004)

2. Bookstein, F.: Principal warps: Thin-plate splines and the decomposition of deformations. IEEE Trans. Pattern Anal. Mach. Intell. 11, 567-585 (1989)

3. Cootes, T., Taylor, C., Cooper, D., Graham, J., et al.: Active shape models-their training and application. Comput. Vis. Image. Und. 61, 38-59 (1995)

4. Deligianni, F., Chung, A., Yang, G.: Nonrigid 2-D/3-D registration for patient specific bronchoscopy simulation with statistical shape modeling: Phantom validation. IEEE Trans. Med. Imag. 25, 1462-1471 (2006)

5. Hutton, T., Buxton, B., Hammond, P., Potts, H.: Estimating average growth trajectories in shape-space using kernel smoothing. IEEE Trans. Med. Imag. 22, 747-753 (2003)

6. Irving, B., Taylor, P., Todd-Pokropek, A.: 3D segmentation of the airway tree using a morphology based method. In: Second International Workshop on Pulmonary Image Analysis, MICCAI (2009)

7. Kaus, M., Pekar, V., Lorenz, C., Truyen, R., Lobregt, S., Weese, J.: Automated 3-D PDM construction from segmented images using deformable models. IEEE Trans. Med. Imag. 22, 1005-1013 (2003)

8. Masters, I., Ware, R., Zimmerman, P., Lovell, B., Wootton, R., Francis, P., Chang, A.: Airway sizes and proportions in children quantified by a video-bronchoscopic technique. BMC Pulmonary Medicine 6, 5-13 (2006)

9. Palágyi, K., Tschirren, J., Hoffman, E., Sonka, M.: Quantitative analysis of pulmonary airway tree structures. Comput. Biol. Med. 36, 974-996 (2006)

10. du Plessis, J., Goussard, P., Andronikou, S., Gie, R., George, R.: Comparing threedimensional volume-rendered CT images with fibreoptic tracheobronchoscopy in the evaluation of airway compression caused by tuberculous lymphadenopathy in children. Pediatr. Radiol. 39, 694-702 (2009)

11. Varma, S., Simon, R.: Bias in error estimation when using cross-validation for model selection. BMC Bioinformatics 7, 1-8 (2006) 